\title{
STIMULUS WITH LIMITED BAND OPTIMIZATION FOR ANALOGUE CIRCUIT TESTING
}

\section{Tomasz Golonek, Piotr Jantos, Jerzy Rutkowski}

Silesian University of Technology, Faculty Of Automatic Control, Electronics And Computer Science, Institute of Electronics, Akademicka 16, 44-100 Gliwice, Poland( $\triangle$ tgolonek@polsl.pl, +48 32 237 2717)

\begin{abstract}
The paper presents an analogue circuit testing method that engages the analysis of the time response to a nonperiodic stimulus specialized for the verification of selected specifications. The decision about the current circuit diagnostic state depends on an amplitude spectrum decomposition of the time response measured during the test. A shape of the test excitation spectrum is optimized with the use of a differential evolution algorithm and it allows for achieving maximum fault coverage and the optimal conditions for fault isolation. Genotypes of the evolutionary system encode the amplitude spectrum of candidates for testing stimuli by means of rectangle frequency windows with amplitudes determined evolutionarily.
\end{abstract}

Keywords: analogue circuit testing, faults isolation, evolutionary computations, amplitude spectrum.

(c) 2012 Polish Academy of Sciences. All rights reserved

\section{Introduction}

Analogue circuit testing and diagnosing are necessary to achieve high quality and reliability of analogue systems and to control their production yield [1-15]. The diversity of analogue signals, noise presence, the limited accuracy of test measurements, circuit parameters' tolerance dispersions and simulation models inaccuracies are exemplary difficulties which are reasons of the complexity of analogue circuit testing [1].

Generally, considering the aim of the approach, the published techniques of analogue circuit testing can be classified into the specification-driven testing (SDT) $[2,6]$ and the fault -driven testing (FDT) $[3,5,7,11]$ groups. The SDT methods are especially desired during tests at the production stage for a validation of design specifications. On the other hand, the FDT concept is proper at the design stage, when precise information (fault location and its identification) can be useable for a project correction. Additionally, the moment of circuit's simulations determines the type of the testing method: with simulations after test (SAT) or with simulations before test (SBT). The simulations can be accepted at the production stage, but it can be necessary for a diagnostic precision improvement running at the test stage lead to a radical increment of the computation time, and it cannot be done at the design stage. The SBT concept is especially suitable if the testing time is critical and the use of the signatures prepared at the before-test stage is possible. Besides, to simplify and improve the efficiency of analogue circuit testing some special modifications of the circuit under test (CUT) can be implemented. This process is called design for testability (DfT). It can improve the access to the test nodes (e.g. boundary scan IEEE 1149.4 , test point selection) $[4,12]$ or it can support a test mode for a CUT (e.g. by its adequate reconnecting) [5].

Evolutionary methods of optimization imitate a natural process of selection and competition in a population [16-19]. The main evolutionary techniques are: genetic algorithm, evolutionary programming, genetic programming, evolutionary strategies and differential evolution. All these methods have some specific properties (e.g. the genotype structure and a 
scheme for genetic operations), but each of them promotes the selection of fitter individuals with higher pressure. Typically, the computational effort for evolutionary approaches is bigger than for classical ones. On the other hand, they have minimal calculating requirements for a considered task (e.g. its limited differentiability is allowed) and they are significantly more resistant to collapsing in its local optimums ( the solving space is sampled in many points coded in numerous populations). These features predispose evolutionary computations for solving especially difficult problems for which deterministic techniques fail [10-13].

The testing method proposed in this paper bases on the concept firstly presented in [13]. This approach can be classified into the SDT and SBT groups and it is especially proper at the production test. The selected CUT specification correctness is verified by controlling energy levels of components extracted from the time response. To assure the possibly highest fault coverage, the amplitude spectrum of a specialized stimulus is optimized evolutionarily in an assumed frequency range by means of differential evolution.

The idea and the motivation for the proposed concept are explained in section 2 . Section 3 contains the description of the evolutionary system. Test results obtained for an example CUT are placed in section 4 and conclusions can be found in section 5 .

\section{Description of the proposed concept}

\subsection{Testing procedure}

The testing stage of the proposed approach is explained in Fig. 1. The time response $y(t)$, obtained for an optimized non-periodic testing excitation $x(t)$, is recorded after a test measuring process and it is used for CUT specification diagnosing.

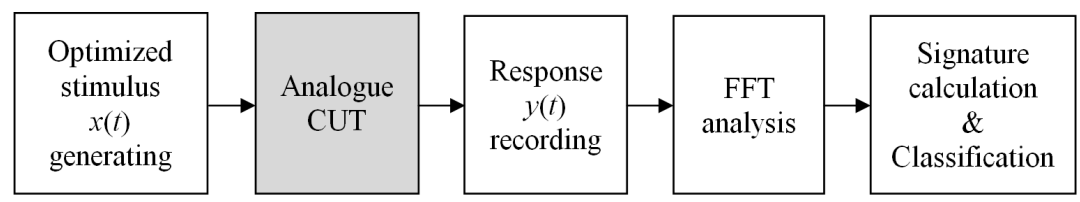

Fig. 1. A testing stage diagram.

Firstly, the spectrum $Y(f)$ of a recorded time response $y(t)$ is determined with the use of Fourier transformation $\mathfrak{I}$ (to reduce the computational time, the FFT algorithm is preferred) for the assumed frequency range $\left\langle f_{m n}, f_{m x}\right\rangle$ :

$$
Y(f)=X(f) \cdot H(f)=\mathfrak{I}\{y(t)\} .
$$

The diagnostic classification depends on an energetic signature $E$ that is calculated as the sum of energies designated for amplitude densities $\left|Y_{p}(f)\right|$ and $\left|Y_{n}(f)\right|$ which are decomposed from a global one $|Y(f)|$ :

$$
E=\int_{f_{m n}}^{f_{m x}}\left(\left|Y_{p}(f)\right|^{2}-\left|Y_{n}(f)\right|^{2}\right) d f=\int_{f_{m n}}^{f_{m x}}\left(\left|W_{p}(f) \cdot Y(f)\right|^{2}-\left|W_{n}(f) \cdot Y(f)\right|^{2}\right) d f=F\left(\mathbf{S}_{i}\right)
$$

The structure of the observed function given above allows to obtain the spectrum $|Y(f)|$ regions which can cause an increase (additive components) or a decrease (subtractive components) of energy $E$ produced by amplitude response dispersions. In effect, appropriate conditions for the faulty states isolation can be achieved more easily. Filtering functions $W_{p}(f)$ and $W_{n}(f)$ are 
created by the $K$ rectangle windows with heights equal to one and with central frequencies determined evolutionarily (see section 3):

$$
\begin{aligned}
& W_{p}(f)=\sum_{k=0}^{K-1} \frac{\operatorname{sign}\left(a_{k}\right)+1}{2} \cdot \Pi\left(\frac{f-f_{k}}{\Delta f_{w d}}\right), \\
& W_{n}(f)=-\sum_{k=0}^{K-1} \frac{\operatorname{sign}\left(a_{k}\right)-1}{2} \cdot \Pi\left(\frac{f-f_{k}}{\Delta f_{w d}}\right),
\end{aligned}
$$

where $a_{k} \in\langle-1,1\rangle$ is a gene assigned for the $k$-th window $\Pi\left(\frac{f-f_{k}}{\Delta f_{w d}}\right)$ with a central frequency $f_{k} \in\left\langle f_{m n}, f_{m x}\right\rangle$ and a width $\Delta f_{w d}$.

The energy value (2) depends on the actual CUT diagnostic state $\mathbf{S}_{i}(i=0, . ., I-1)$. So, the identification of a state $\mathbf{S}_{i}$ can be achieved after classifying signature $E$ to the adequate ambiguity set $\mathbf{C}_{i}$ stored in a faults dictionary. In case of a production test, faulty circuit detection is the most important and, typically, only two states are considered $(I=2)$ then. During this kind of test (GO/NO GO), healthy CUTs (i.e. with acceptable values for all of the validated specifications) should be classified to the set $\mathbf{S}_{0}$, and to the set $\mathbf{S}_{1}$ otherwise (i.e. with an unacceptable value for one or more of the validated specifications). The boundary values of all signatures $\mathbf{C}_{i}$ can be determined after a Monte Carlo analysis run for all states $\mathbf{S}_{i}$ during the before-test stage.

Of course, the signatures (2) decomposition depends strongly on the shapes of the testing excitation and filtering functions spectra, too. Their optimization allows to reach the most appropriate conditions for an analogue faults isolation. In the diagnostic system described, the functions $|X(f)|, W_{p}(f)$ and $W_{n}(f)$ are determined by means of differential evolution. The optimization process allows to maximize the quality factor $Q$ for a training set that contains $L$ random patterns of amplitude responses for each diagnostic state $\mathbf{S}_{i}$ considered for isolation.

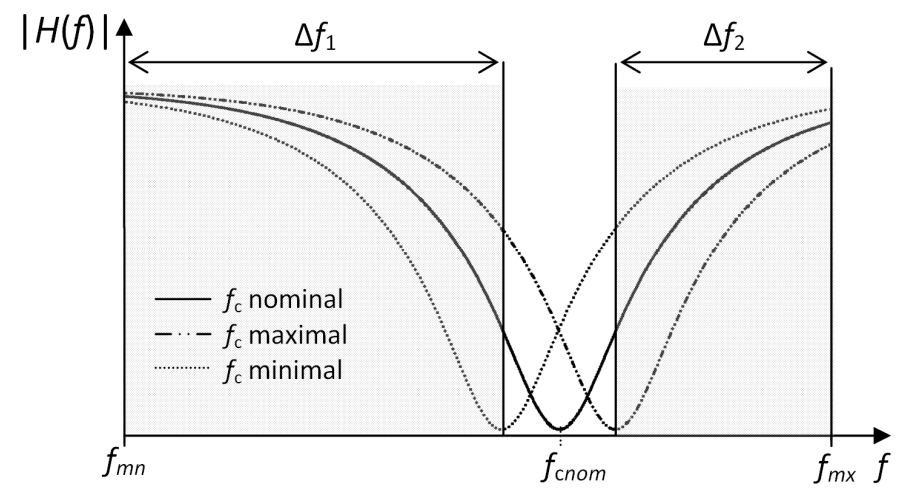

Fig. 2. An exemplary amplitude response.

The motivation of spectrum $|X(f)|$ optimization is explained in Fig. 2. There are three amplitude responses for a band-stop filter with: central frequencies $f_{c}$ placed in a nominal $f_{\text {cnom }}$ and in assumed allowed boundary minimal and maximal positions respectively. If only this frequency $f_{c}$ should be examined during the testing procedure, the testing excitation $x(t)$ should have the amplitude spectrum $|X(f)|$ near to the illustrated two rectangle windows with widths $\Delta f_{1}$ and $\Delta f_{2}$. This kind of stimulus allows to assure a practically still value of response $y(t)$ energy for healthy CUTs and then a faulty circuits isolation will be able to be reached more easily. 


\subsection{Initial assumptions}

Before determining the testing signal $x(t)$, some initial steps are necessary. Firstly, the range $f_{k} \in\left\langle f_{m n}, f_{m x}\right\rangle$, and the number $M$ of points for $\mathrm{AC}$ analysis have to be assumed. Besides, the set $\mathbf{P}$ of the $N$ observed CUT's parameters (e.g. cut-off frequencies, ripple values, minimal attenuation) has to be defined:

$$
\mathbf{P}=\left\{p_{0}, . ., p_{N-1}\right\} .
$$

Next, the set $\mathbf{D}$ of maximal allowed lower and upper deviations for all evaluated specifications from (4) can be assumed:

$$
\mathbf{D}=\left\{\begin{array}{ll}
p_{0}^{m x} & p_{N-1}^{m x} \\
p_{0}^{m n}, . . & p_{N-1}^{m n}
\end{array}\right\} .
$$

After the classification process, the diagnostic system should detect a healthy CUT state $\mathbf{S}_{0}$ if all parameters $p_{n}(n=1,2, . ., N)$ are within their allowed ranges $\left\langle p_{n}^{m n}, p_{n}^{m x}\right\rangle$ and a faulty state otherwise. In the next step, simulations with a Monte Carlo AC analysis can be executed and a set of training patterns for $I$ diagnostic states provided for isolation can be prepared. Finally, the following parameters for an evolutionary system have to be assumed:

- the number of frequency windows $K$,

- the quantity of population $U$,

- the number of generations $V$,

- reproduction weights $\eta, \kappa$.

\section{Evolutionary optimization system}

\subsection{Phenotype coding}

As mentioned in the recent section, an applied stimulus $x(t)$ has an amplitude spectrum $|X(f)|$ created by $K$ rectangle windows spaced in the assumed range $\left\langle f_{m n}, f_{m x}\right\rangle$ :

$$
|X(f)| \approx \sum_{k=0}^{K-1}\left|a_{k}\right| \cdot \Pi\left(\frac{f-f_{k}}{\Delta f_{w d}}\right) .
$$

The widths $\Delta f_{w d}$ for all $K$ windows are the same and they are equal to:

$$
\Delta f_{w d}=\frac{f_{m x}-f_{m n}}{K} .
$$

The value $\Delta f_{w d}$ can be interpreted as a resolution of frequency windowing, a wider components creation is possible by collecting a series of windows. The proposed construction of the spectrum $|X(f)|$ allows to stimulate a CUT in considered regions of its frequency response (in area of cut-off frequencies, evaluated ripples etc.). Practically, only some approximations of the rectangle windows (6) of a signal $x(t)$ amplitude spectrum are reachable (the main difficulties are: the presence of the Gibbs effect, limited time of stimulation, limited resolution of IDFT), but usually these distortions represent only a small part of the total energy calculated for a frequency window. Besides, the optimization system can be easily modified to calculate the signature (2) after reconstruction of density $X(f)$ from a practical 
signal $x(t)$ for that duration time and resolution of generation were limited. This allows to minimize the influence of practical restrictions for the system efficiency.

The differential evolution [16] algorithm is searching for: the optimal gain coefficients $\left|a_{k}\right|$ for $K$ windows and the optimal filtering functions $W_{p}(f)$ and $W_{n}(f)$ from equation (2). The structure of the $v$-th generation $\mathbf{G}^{(v)}$ is presented in (8):

$$
\mathbf{G}^{(v)}=\left[\begin{array}{ccccc}
a_{0}^{(v)(0)} & a_{1}^{(v)(0)} & \ldots & a_{K-2}^{(v)(0)} & a_{K-1}^{(v)(0)} \\
a_{0}^{(v)(1)} & a_{1}^{(v)(1)} & \ldots & a_{K-2}^{(v)(1)} & a_{K-1}^{(v)(1)} \\
\vdots & \vdots & \vdots & \vdots & \vdots \\
a_{0}^{(v)(U-2)} & a_{1}^{(v)(U-2)} & \ldots & a_{K-2}^{(v)(U-2)} & a_{K-1}^{(v)(U-2)} \\
a_{0}^{(v)(U-1)} & a_{1}^{(v)(U-1)} & \ldots & a_{K-2}^{(v)(U-1)} & a_{K-1}^{(v)(U-1)}
\end{array}\right]
$$
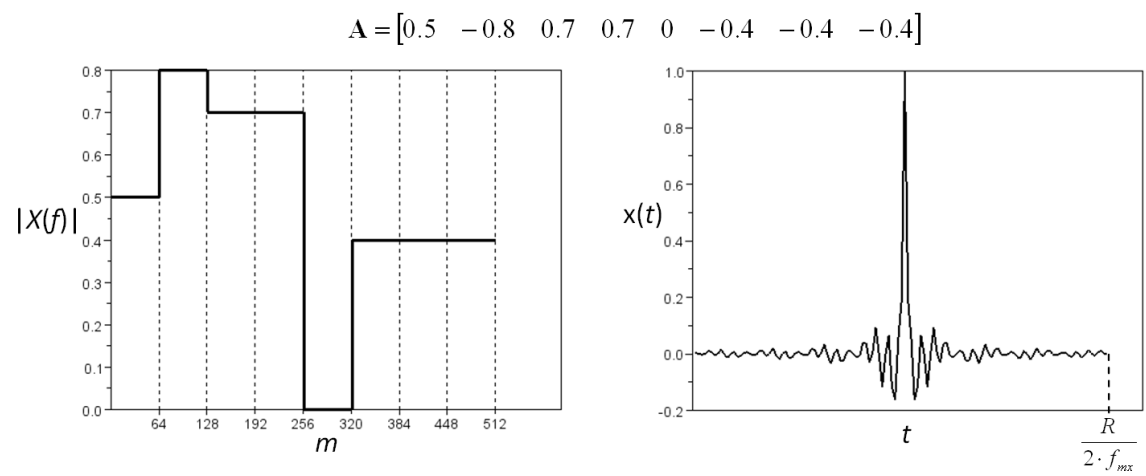

Fig. 3. An amplitude spectrum $|X(f)|$ and time waveform $x(t)$ decoded from an exemplary genotype A.
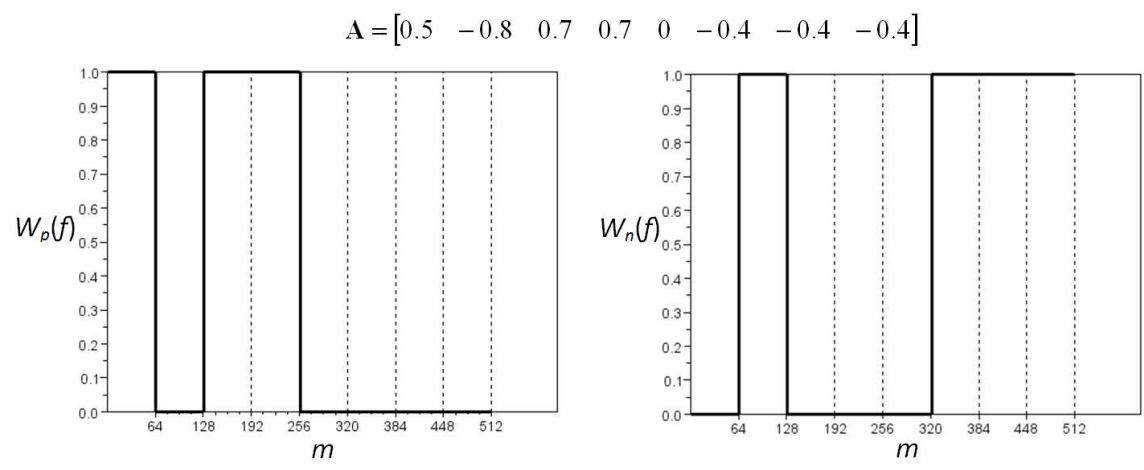

Fig. 4. Filtering functions $W_{p}(f)$ and $W_{n}(f)$ decoded from an exemplary genotype $\mathbf{A}$.

Genotypes contain real numbered coefficients, their absolute values code heights of windows for testing the stimulus spectrum $|X(f)|$ and their signs code shapes of filtering functions $W_{p}(f)$ and $W_{n}(f)$. For illustration of the coding method, resultant phenotypes decoded from an exemplary genotype A for $K=8$ windows and $M=512$ frequency steps are presented in Fig. 3 and Fig. 4. Additionally, there is an interpolated waveform of stimuli constructed for $R$ samples acquired for Nyquist rate and $f_{m n}=0$. 


\subsection{Fitness function}

The qualities of phenotypes are evaluated basing on the fitness function $Q$ that reaches the maximal value for the best genotype $\mathbf{A}_{b t}$ found during $U$ evolutionary cycles. The adequate construction of this function is very important for evolutionary systems [16-19] and in the tested implementation, the weighted sum of three components has been applied:

$$
Q=\frac{\alpha}{L \cdot I}+\frac{\beta}{L \cdot I \cdot M}+\frac{\gamma}{L \cdot I \cdot M^{2}},
$$

where $\alpha, \beta, \gamma$ are the components proportional to the number of isolated CUT states, to the minimal and to the average distance between separated states from a training set respectively:

$$
\begin{aligned}
& \alpha=\sum_{i} \sum_{l} \operatorname{count}\left(\mathbf{S}_{i l} \in \mathbf{C}_{i}, \mathbf{S}_{i l} \notin \mathbf{C}_{j}\right), \\
& \beta=\min \left[\underset{\mathbf{S}_{i l} \in \mathbf{C}_{i}, \mathbf{S}_{j l} \in \mathbf{C}_{j}}{\forall} \operatorname{dist}\left(\mathbf{S}_{i l}, \mathbf{S}_{j l}\right)\right], \\
& \gamma=\operatorname{mean}\left[\underset{\mathbf{S}_{i l} \in \mathbf{C}_{i}, \mathbf{S}_{j l} \in \mathbf{C}_{j}}{\forall} \operatorname{dist}\left(\mathbf{S}_{i l}, \mathbf{S}_{j l}\right)\right], \\
& \operatorname{dist}\left(\mathbf{S}_{i l}, \mathbf{S}_{j l}\right)=\left|E\left(\mathbf{S}_{i l}\right)-E\left(\mathbf{S}_{j l}\right)\right|, \\
& j \neq i, i=0, . ., I-1, j=0, . ., I-1, l=0, . ., L-1 .
\end{aligned}
$$

The proposed values of weights from function $Q$ allow to obtain a hierarchical optimization for that the most important is the level of fault coverage, however the energetic distances between isolated states are optimized with lower priorities.

\subsection{Differential evolution algorithm}

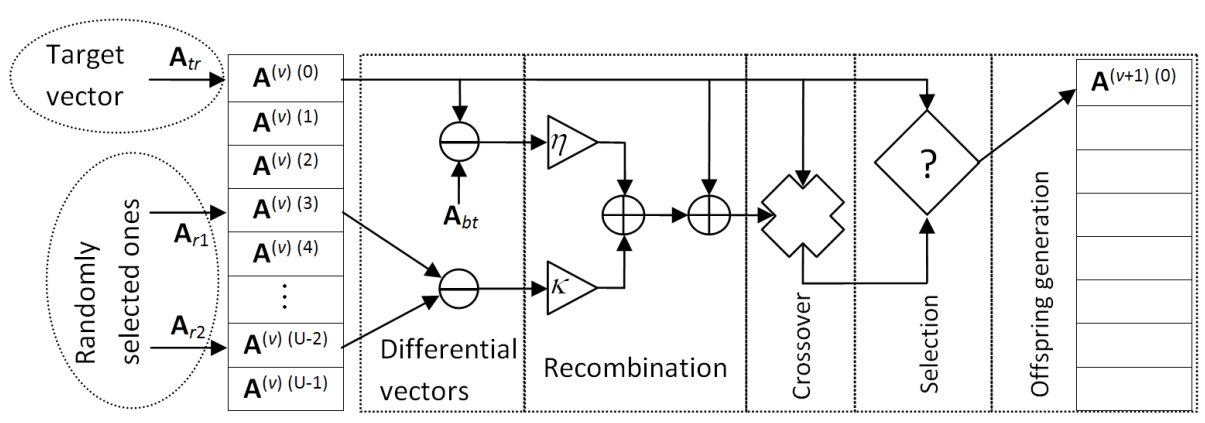

Fig. 5. Differential evolution scheme.

The first generation of the evolutionary system is built randomly with a uniform distribution of probability. All $U$ genotypes are collected by real numbered genes selected from a normalized range $a_{k} \in\langle-1,1\rangle$. After the fitness $Q$ calculation for all primary individuals, the evolutionary process of the new offspring creation starts. The implemented optimization system uses the scheme illustrated in Fig. 5. Each evolutionary cycle is executed for the target vector $\mathbf{A}_{t r}$ (the first one from a parent population in the illustrated case). At the beginning, two individuals $\mathbf{A}_{r 1}$ and $\mathbf{A}_{r 2}$ are selected randomly and they create a first differential component that assures mutation of the target one with weight $\kappa$. To preserve the best current solution $\mathbf{A}_{b t}$, the second differential vector is calculated basing on it with the 
weight $\eta$. Next, the intermediate individual $\mathbf{A}_{\text {new }}$ is calculated from differential components and from target one adequately to the equation:

$$
\mathbf{A}_{\text {new }}=\mathbf{A}_{t r}+\eta \cdot\left(\mathbf{A}_{b t}-\mathbf{A}_{t r}\right)+\kappa \cdot\left(\mathbf{A}_{r 1}-\mathbf{A}_{r 2}\right) .
$$

Then, $\mathbf{A}_{\text {new }}$ vector is crossed with the target one and an offspring individual is created. In the tested system, during the crossover procedure, genes randomly selected in $\mathbf{A}_{t r}$ with probability 0.5 are replaced by average values calculated between adequate genes from $\mathbf{A}_{n e w}$ and $\mathbf{A}_{t r}$ ones. Finally, the genotype with the better fitness value $Q$ (a child or a target one) is inserted to the offspring generation. The described procedure is repeated $U$ times and then the period of the new population creating is closed. The evolutionary system stops work after producing $V$ generations and the best individual $\mathbf{A}_{b t}$ found finally codes the optimal phenotype.

\section{Computational example}

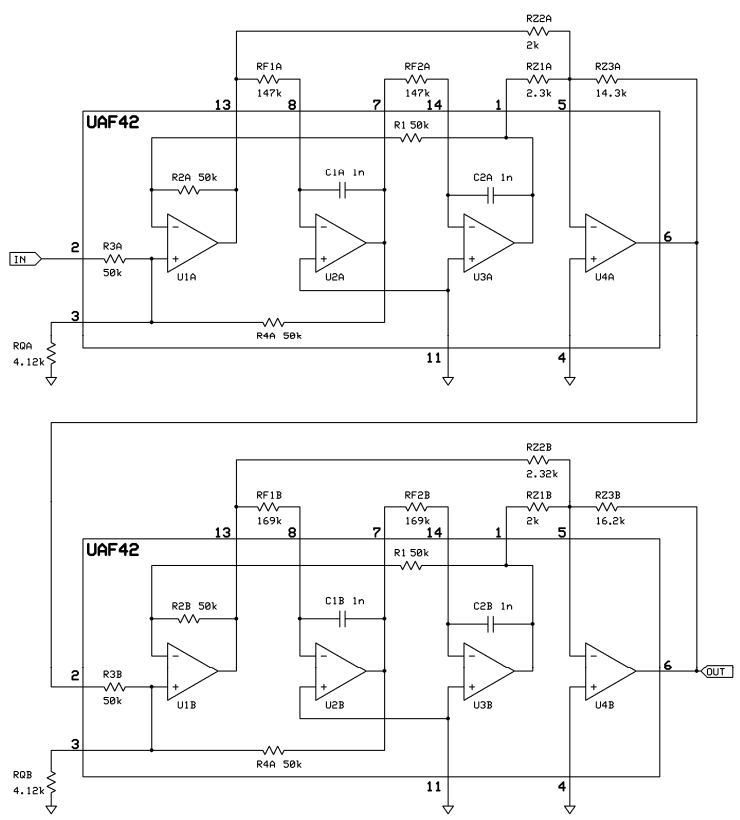

Fig. 6. Stop-band 4-th order Butterworth filter.

The filter selected for the example CUT is in Fig. 6. This is a stop-band filter designed in the Burr-Brown UAF42 Filter Design Program (Filter42) [20] for the following specifications:

- Butterworth filter,

- order equal to 4 ,

- notch frequency $1 \mathrm{kHz}$,

- bandwidth $0.2 \mathrm{kHz}$.

Firstly, a Monte Carlo AC analysis of the CUT was executed for boundary frequencies $f_{m n}=512 \mathrm{~Hz}$ and $f_{m x}=1536 \mathrm{~Hz}$ and for $M=512$ points, so the analyzed step was equal to:

$$
\Delta f_{a c}=\frac{f_{m x}-f_{m n}}{M}=2 \mathrm{~Hz} .
$$


The set $\mathbf{P}$ of $N=6$ CUT's tested specifications (below) was defined:

$$
\begin{aligned}
& p_{0}=\left|H\left(f_{m n}\right)\right|, p_{1}=|H(1 \mathrm{kHz})|, p_{2}=f_{c}, \\
& p_{3}=\Delta f_{o f f}, p_{4}=\varepsilon_{p b m x}, p_{5}=\varepsilon_{r b m x},
\end{aligned}
$$

where $p_{0}, p_{1}, p_{2}, p_{3}, p_{4}$ and $p_{5}$ are: the amplitude response for the lowest analyzed frequency $\left|H\left(f_{m n}\right)\right|$, the attenuation value for a nominal notch frequency $|H(1 \mathrm{kHz})|$, a reject band central frequency position $f_{c}$ (calculated as the mean value between lower and upper cut-off frequencies), the reject band width $\Delta f_{\text {off }}$, and values for maximal gains: in the pass band $\varepsilon_{p b m x}$ and in the reject band $\varepsilon_{r b m x}$ respectively. The allowed ranges for all considered specifications $\mathbf{P}$ were assumed as follows:

$$
\mathbf{D}=\left\{\begin{array}{l}
p_{0}^{m x}=1.2 \frac{\mathrm{V}}{\mathrm{V}} p_{1}^{m x}=-32 \mathrm{~dB}, p_{2}^{m x}=1.2 \mathrm{kHz} p_{3}^{m x}=240 \mathrm{~Hz} p_{4}^{m x}=3 \mathrm{~dB} p_{5}^{m x}=-20 \mathrm{~dB} \\
p_{0}^{m n}=0.8 \frac{\mathrm{V}}{\mathrm{V}} p_{1}^{m n}=-\infty, p_{2}^{m n}=0.8 \mathrm{kHz} p_{3}^{m n}=160 \mathrm{~Hz} \quad p_{4}^{m n}=0, p_{5}^{m n}=-\infty
\end{array}\right\} .
$$

The CUT should be classified to a healthy state $\mathbf{S}_{0}$ if all tested specifications are within their allowed ranges (14), or to a faulty state $\mathbf{S}_{1}$ otherwise.

Next, a training set for a realization of the GO/NO GO production test can be prepared. This set consists of $100 \mathrm{AC}$ simulation results (amplitude responses) for the CUTs with state $\mathbf{S}_{0}$ and 100 results for CUTs with state $\mathbf{S}_{1}$. To obtain the CUT's specifications destruction, all resistors and capacitors from the circuit were deviated randomly from their nominal values up to $\pm 8 \%$ with uniform distribution. Training patterns generated for the two considered states are illustrated in Fig. 7.

a)

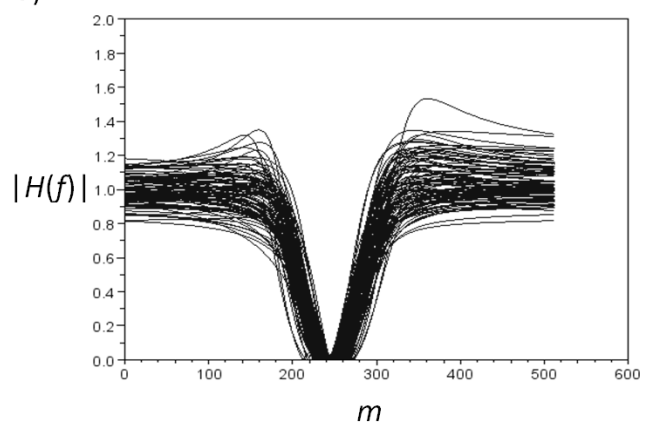

b)

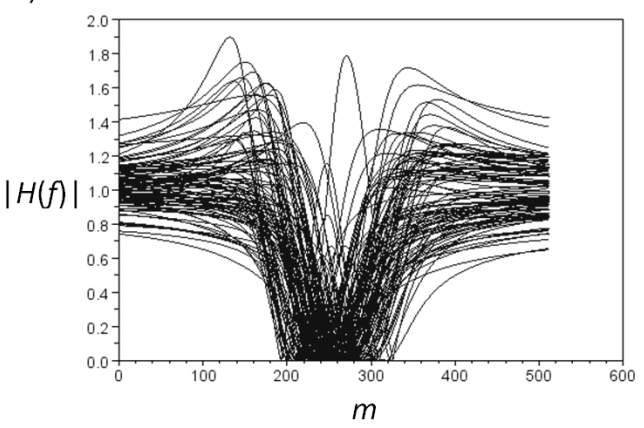

Fig. 7. Amplitude responses from the training set: a) healthy CUTs, b) faulty ones.

The evolutionary system was started with the following parameters:

- the number of frequency windows $K=16$,

- the quantity of population $U=100$,

- the number of generations $V=200$,

- reproduction weights $\eta=0.8, \kappa=0.3$.

The best genotype:

$$
\begin{aligned}
& \mathbf{A}_{b t}(0, . ., 7)=\left[\begin{array}{llllllll}
-0.072 & -0.193 & -0.017 & 0.159 & 0.046 & -0.006 & 0.316 & -0.722
\end{array}\right], \\
& \mathbf{A}_{b t}(8, . ., 15)=\left[\begin{array}{llllllll}
-0.253 & 0.489 & 0.488 & -0.414 & 0.529 & 0.374 & -0.209 & -0.567
\end{array}\right] \text {, }
\end{aligned}
$$


was created in the 195th generation and it coded the phenotype which allowed to isolate states from the training set with efficiencies presented in Table 1. Additionally, to evaluate the generalization level, there are also detection results added for diagnostic states which were not considered during the optimization process (validation set).

Table 1. Results obtained for the best found stimuli.

\begin{tabular}{|c|c|c||c|c||}
\hline Type of set & $\begin{array}{c}\text { Faulty states correct } \\
\text { detections, } \%\end{array}$ & $\begin{array}{c}\text { Faulty states detected } \\
\text { as healthy, } \%\end{array}$ & $\begin{array}{c}\text { Healthy states correct } \\
\text { detections, } \%\end{array}$ & $\begin{array}{c}\text { Healthy states detected } \\
\text { as faulty, } \%\end{array}$ \\
\hline \hline Training & 74 & 26 & 100 & 0 \\
\hline Validation & 67 & 33 & 92 & 8 \\
\hline
\end{tabular}

The shapes of the specialized stimulus amplitude spectrum $|X(f)|$ in the assumed range $\left\langle f_{m n}, f_{m x}\right\rangle$ and normalized time waveform $x(t)$ obtained after IDFT calculation are presented in Fig. 8.
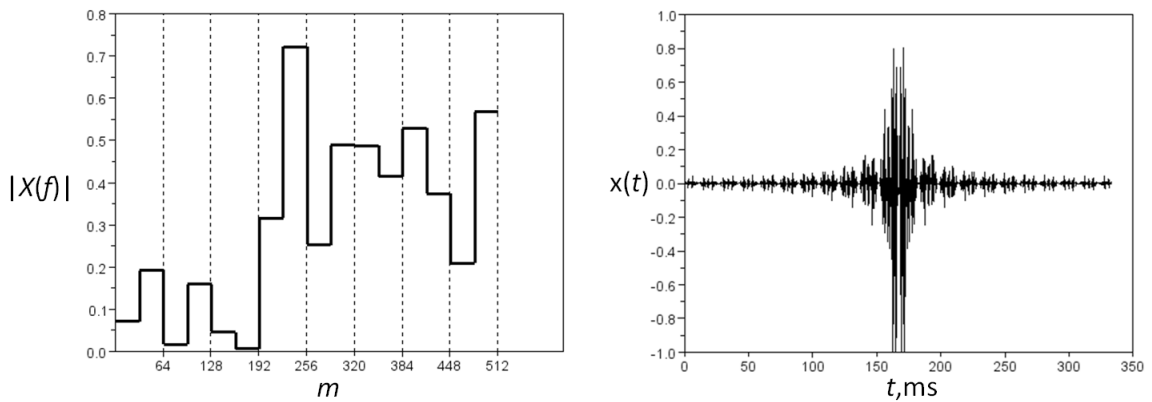

Fig. 8. The amplitude spectrum $|X(f)|$ and time waveform $x(t)$ decoded from the best genotype $\mathbf{A}_{b t}$.

Of course, before the IDFT calculation, the amplitude spectrum $|X(f)|$ has to be shifted adequately to the minimal frequency $f_{m n}$. The number of points which have to be added at the beginning can be calculated from:

$$
\Delta M=\operatorname{round}\left(\frac{f_{m n}}{\Delta f_{a c}}\right),
$$

and for the example circuit, the shift is equal to 256 points. The optimal filtering functions $W_{p}(f)$ and $W_{n}(f)$ obtained are shown in Fig. 9.
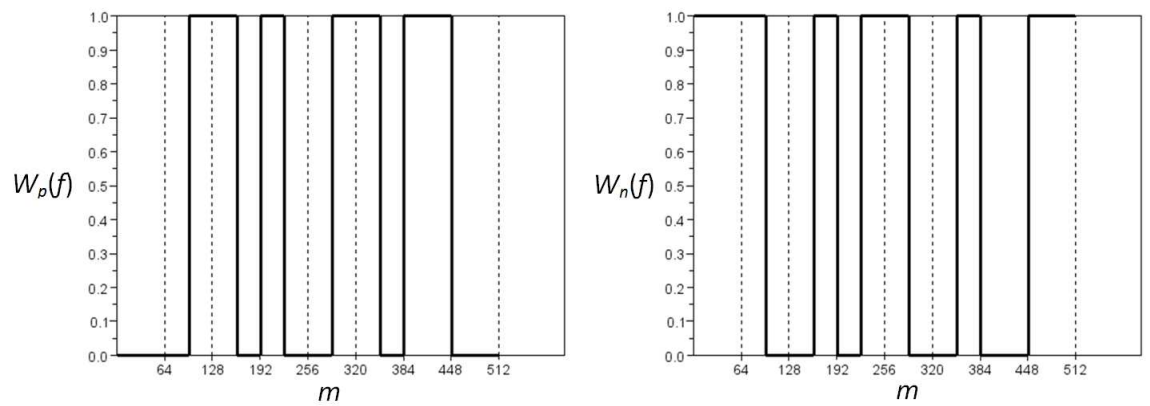

Fig. 9. Filtering functions $W_{p}(f)$ and $W_{n}(f)$ decoded from the best genotype $\mathbf{A}_{b t}$. 
The achieved results of faulty CUTs separation (Table 1) are not very high, but it should be emphasized that for the reference stimulus (sine cardinal):

$$
\mathrm{x}_{\text {ref }}(t)=\operatorname{sinc}(2 \cdot \pi \cdot 1536 \cdot t)
$$

with constant level of its amplitude spectrum in the considered testing range, the training faulty states separation is only about $7 \%$, so a $67 \%$ of improvement was reached. Besides, some part of patterns from the training set has specifications $\mathbf{P}$ lying near to the allowed ones D, so their isolation is very difficult. Additionally, the found stimuli performance improvement can be easily noticed in Fig. 10.

a)

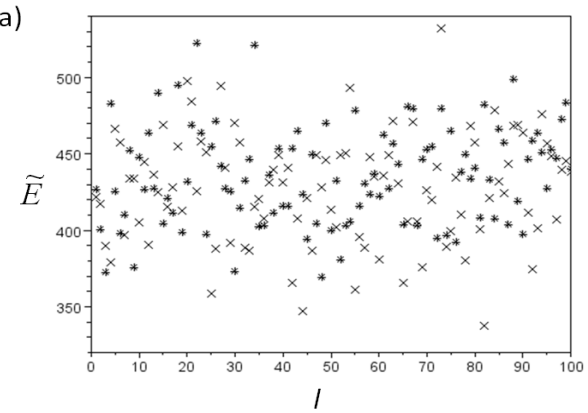

b)

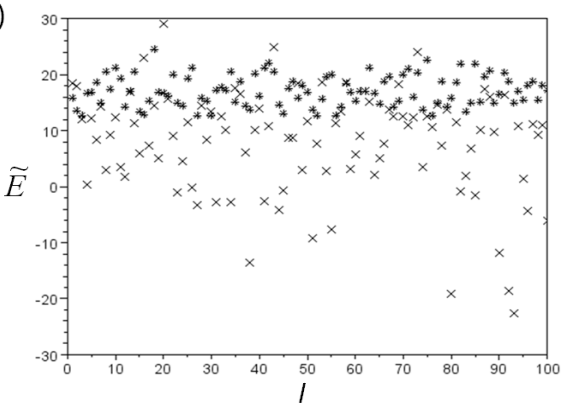

Fig. 10. Signatures decomposition for the patterns from training set: a) without, b) with optimization.

There are two decompositions of estimated values (calculated as a subtraction of absolute values of components instead of their square values for time saving) of the $L$ signatures (2) presented there, obtained for the considered training set (asterisks for $\mathbf{S}_{0}$, crosses for $\mathbf{S}_{1}$ patterns) by reference stimuli $x_{\text {ref }}(t)$ and the best one found $x(t)$. It is obvious that testing excitation $x(t)$ specialized after evolutionary optimization has given significantly better conditions for a diagnostic classification. The decomposition obtained for the non-optimized signal is chaotic, so its grouping is impossible.

The achievable accuracy for practical stimuli can be evaluated basing on DFT results illustrated in Fig. 11, that were calculated for $R=1024$ samples of the found waveform $x(t)$. As can be seen, after shifting the frequency, the reconstructed shape of the spectrum is very close to the theoretical one shown in Fig. 8.

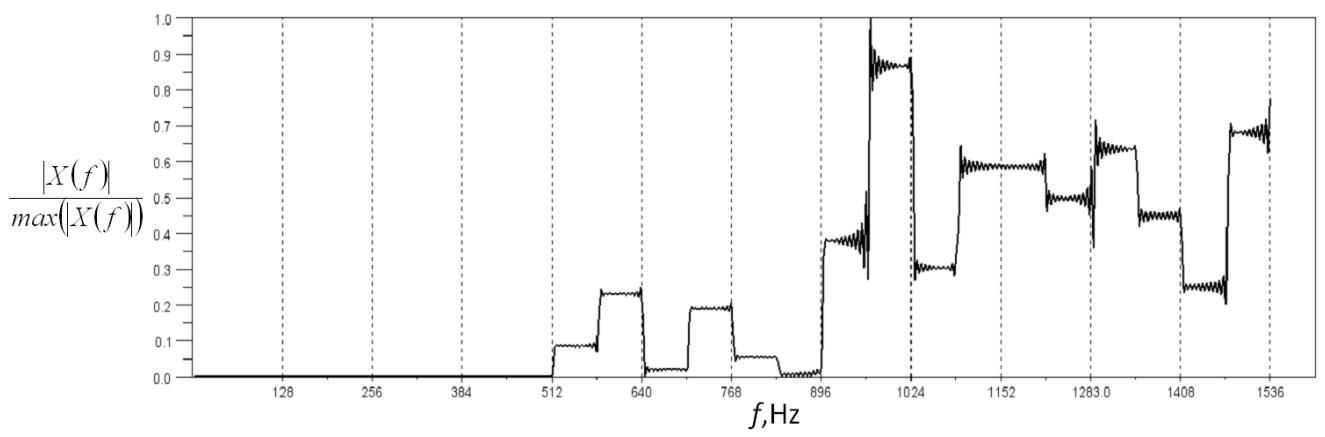

Fig. 11. The normalized amplitude spectrum of the testing signal $x(t)$. 


\section{Conclusions}

The method presented in this paper allows finding a testing excitation optimized for the validation of specifications of analogue circuits. This concept is especially suitable for a quick production test for the testing time has to be minimal. The described approach needs only one response analysis recorded at the CUT output after its excitation. As was proven in the computational example, a stimulus spectrum evolutionary optimization gives a significant improvement of its diagnostic usefulness. The proposed signature is a sum of energetic components calculated for an optimally determined frequency window located in the CUT spectrum. In practice, this signature can be obtained after a FFT analysis of the measured response. Alternatively, the necessary components can be determined from an observed signal in the time domain by means of convolution calculation with the masks designed for special digital filters with amplitude responses adequate to the filtering functions $W_{p}(f)$ and $W_{n}(f)$. In the future, the simple linear classification applied in the tested system can be replaced by a more efficient one and it should increase the faults coverage. Besides, the training set can be additionally extended to improve the generalization level.

\section{References}

[1] Milor, L.S. (1998). A Tutorial Introduction to Research on Analog and Mixed-Signal Circuit Testing. IEEE Trans. on Cir. and Syst.-II, Analog and Digital Signals Processing, 45(10), 1389-1407.

[2] Załęski, D., Zielonko, R., Bartosiński, B. (2010). Application of Complementary Signals in Built-In Self Testers for Mixed-Signal Embedded Electronic Systems. IEEE Trans. on Inst. and Measure., 59(2), 345-352.

[3] Tadeusiewicz, M., Hałgas, S. (2009). Multiple catastrophic fault diagnosis of linear circuits considering the component tolerances. In Proc. ECCTD, 647-650.

[4] Bartosiński, B., Toczek, W. (2003). Some methods of diagnosis of analog circuits using mixed signal test bus IEEE 1149.4. Metrology and Measurement Systems, 10(2), 157-172.

[5] Chalk, C., Zwoliński, M. (1997). A Design for Test Technique to Increase the Resolution of Analogue Supply Current Tests. Electronic Letters, 33(21).

[6] Jantos, P., Golonek, T., Rutkowski, J. (2011). An Analogue Electronic Circuits Specification Driven Testing with the use of Time Domain Response's Features. In Proc. Mixed Design of Integrated Circuits and Systems, MIXDES.

[7] Jantos, P., Grzechca, D., Rutkowski, J. (2009). Global Parametric Faults Identification in Analogue Electronic Circuits. Metrology and Measurement Systems, 16(3), 391-402.

[8] Alippi, C., Catelani, M., Fort, A., Mugnaini, M. (2005). Automated Selection of Test Frequencies for Fault Diagnosis in Analog Electronic Circuits. IEEE Trans. On Instr. and Measur., 54(3).

[9] Tadeusiewicz, M., Hałgas, S. (2010). A method for fast simulation of multiple catastrophic faults in analogue circuits. International Journal of Circuit Theory and Applications, 38(3), 275-290.

[10] Jantos, P., Grzechca, D., Rutkowski, J. (2010). An analogue integrated circuits yield optimisation with the use of genetic algorithm. In Proc. International Conference on Signal and Electronic Systems, ICSES, 293-296.

[11] Korzybski, M. (2008). Dictionary method for multiple soft and catastrophic fault diagnosis based on evolutionary computation. In Proc. International Conference on Signal and Electronic Systems, 553-556.

[12] Golonek, T., Rutkowski, J. (2007). Genetic-Algorithm-Based Method for Optimal Analog Test Points Selection. IEEE Trans. on Cir. and Syst.-II., 54(2), 117-121.

[13] Golonek, T., Jantos, P., Rutkowski, J. (2011). The Use of Stimulus with Limited Band for Analogue Circuit Testing. The National Electronics Conference, 884-889. (in Polish)

[14] Grzechca, D. (2011). Soft Fault Clustering in Analog Electronic Circuits with the Use of Self Organizing Neural Network. Metrology and Measurement Systems, 18(4), 555-568. 
[15] Pułka, A. (2011). Two Heuristic Algorithms for Test Point Selection in Analog Circuits Diagnoses. Metrology and Measurement Systems, 18(1), 115-128.

[16] Price, K., Storn, R.M., Lampinen, J.A. (2005). Differential Evolution - A Practical Approach to Global Optimization. Springer.

[17] Goldberg, D.E. (1989). Genetic Algorithms in Search / Optimization and Machine Learning. Addison Wesley.

[18] Michalewicz, Z. (1996). Genetic Algorithms+Data Structures=Evolution Programs. Springer-Verlag.

[19] Koza, J.R. (1992). Genetic Programming: on the programming of computers by means of natural selection. MIT Press.

[20] UAF42 design software. http://focus.ti.com/docs/prod/folders/print/uaf42.html. 